\title{
A retrospective study on Lindley distribution
}

\begin{abstract}
Generalizations make a distribution more flexible especially for studying the tail properties. There exist many generalized family of continuous univariate distributions. In this article, a survey on Lindley distribution, its extensions and classes is conducted. Several available generalizations of the distribution are reviewed and recent trends in the construction of generalized classes are discussed.
\end{abstract}

Keywords: generalizations, Lindley distribution, power distribution, truncated distributions, wrapped distributions
Volume 7 Issue 3 - 2018

\section{Lishamol Tomy}

Department of Statistics, Deva Matha College, India

Correspondence: Lishamol Tomy, Department of Statistics, Deva Matha College, Kuravilangad, Kerala, 686633, India, Email lishatomy@gmail.com

Received: February 15, 2018 | Published: April 03, 2018

\section{Introduction}

The analysis and modeling of lifetime data are very important in applied sciences such as engineering, public health, actuarial science, biomedical studies, demography, industrial reliability and other applied sciences. Therefore, it seems crucial to find statistical distributions for model real-world phenomena. There are a number of lifetime distributions in statistical literature including exponential, Weibull, gamma and lognormal distributions. The Weibull distribution is one of the most popular and widely used models in life testing and reliability theory. ${ }^{1}$ Introduced a one-parameter distribution known as Lindley distribution. This model is used as an alternative model for existing statistical distributions. Lindley distribution has several real applications where the data show the non-monotone shape for their hazard rate.

Generalizations of existing distribution is an another interest in statistical research. The extended distributions have attracted in statistical literature to develop new models. The transformations of distributions have been proved useful in exploring skewness and tail properties, and also for improving the goodness-of-fit of the extended family. The Lindley distribution has been generalized by many authors in recent years. This study concentrates on conducting an extensive enquiry on those different fields of existing knowledge.

This paper is organized as follows. Section 2 deals with the Lindley Distribution. Section 3 discusses existing generalizations of Lindley distribution. Finally, concluding remarks are given in Section 4.

\section{Lindley distribution}

The probability density function (pdf) of one parameter Lindley distribution is given by

$$
f(x ; \theta)=\frac{\theta^{2}}{1+\theta}(1+x) e^{-\theta x} ; x>0, \theta>0
$$

The cumulative density function (cdf) of one parameter Lindley distribution, corresponding to the pdf given in equation (2.1) is

$$
F(x ; \theta)=1-\left[1+\frac{\theta x}{\theta+1}\right] e^{-\theta x} ; x>0, \theta>0
$$

This distribution is derived as a mixture of exponential $(\theta)$ and Gamma $(2, \theta)$ distribution.

Hence the pdf takes the alternate form,

$$
f(x ; \theta)=p f_{1}(x)+(1-p) f_{2}(x)
$$

where $\beta=\frac{\theta}{1+\theta}, f_{1}(x)=\theta e^{-\theta x}, f_{2}(x)=\frac{\theta^{2}}{\Gamma(2)} e^{-\theta x} x^{2-1}$

$\mathrm{and}^{2}$ proved that in modeling and analysis of lifetime data, Lindley distribution provides a better model in many ways than the very usual exponential distribution. Along with various other properties they derived the central moments of Lindley distribution as,

$$
\begin{gathered}
\mu_{2}=\frac{\theta^{2}+4 \theta+2}{\theta^{2}(\theta+1)^{2}} \\
\mu_{3}=\frac{2\left(\theta^{3}+6 \theta^{2}+6 \theta+2\right)}{\theta^{3}(\theta+1)^{3}} \text { and } \\
\mu_{4}=\frac{3\left(3 \theta^{4}+24 \theta^{3}+44 \theta^{2}+32 \theta+8\right)}{\theta^{4}(\theta+1)^{4}}
\end{gathered}
$$

Mazucheli ${ }^{3}$ discussed the applications of Lindley distribution on lifetime data regarding competing risks. Alternately, Shanker et al. ${ }^{4}$ made a comparison study of the goodness of fit of exponential and Lindley distributions on modeling of lifetime data. They provide different graphs for pdfs and cdfs for the same values of parameter for a visual comparison on the nature of the two distributions. They provide different associated functions as presented in Table 1.

The study associated fifteen different data sets and the fact revealed is that in some cases exponential distribution provides better fit than the Lindley distribution whereas in other cases Lindley distribution provides better fit than the exponential distribution. Thus ${ }^{4}$ does not made a final conclusion on the superiority of the two distributions. They arrive at a statement that the suitability depends on the nature of data. Exponential is simple, still Lindley is more flexible.

\section{Quasi Lindley distribution}

Shanker ${ }^{5}$ introduced Quasi Lindley distribution (QLD). QLD with parameters $\alpha$ and $\theta$ is defined by its pdf

$$
f(x, \alpha, \theta)=\frac{\theta(\alpha+x \theta)}{\alpha+1} e^{-\theta x}, x>0, \theta>0, \alpha>-1
$$

It can be easily seen that at $\alpha=\theta$, the QLD equation (2.3) reduces to the Lindley distribution given by equation (2.1) and at $\alpha=0$, it reduces to the gamma distribution with parameters $(2, \theta)$. The pdf equation (2.3) can be shown as a mixture of exponential $(\theta)$ and gamma $(2, \theta)$ distributions as follows:

$$
f(x, \alpha, \theta)=p f_{1}(x)+(1-p) f_{2}(x)
$$


where $p=\frac{\alpha}{\alpha+1}, f_{1}(x)=\theta e^{-\theta x}$ and $f_{2}(x)=\theta^{2} x e^{-\theta x}$ The cdf of the QLD is obtained as

$$
F(x, \alpha, \theta)=1-\frac{1+\alpha+\theta x}{\alpha+1} e^{-\theta x}, x>0, \theta>0, \alpha>-1
$$

The QLD has been fitted to a number of data sets to which earlier the Lindley distribution has been fitted by others and it was found that to almost all these data-sets, the QLD provides closer fits than those by the Lindley distribution.

Table I Different measures of exponential and Lindley distributions

\begin{tabular}{lcc}
\hline Measure & $\begin{array}{l}\text { Exponential } \\
\text { distribution }\end{array}$ & Lindley distribution \\
\hline coefficient of variation & । & $\sqrt{\frac{\theta^{2}+4 \theta+2}{\theta+2}}$ \\
coefficient of Skewness $\left(\sqrt{\beta_{1}}\right)$ & 2 & $\frac{2\left(\theta^{3}+6 \theta^{2}+6 \theta+2\right)}{\left(\theta^{2}+4 \theta+2\right)^{3 / 2}}$ \\
coefficient of Kurtosis $\left(\beta_{2}\right)$ & 9 & $\frac{3\left(3 \theta^{4}+24 \theta^{3}+44 \theta^{2}+32 \theta+8\right)}{\left(\theta^{2}+4 \theta+2\right)^{2}}$ \\
Index of dispersion, $\gamma=\frac{\mu_{2}}{\mu_{1}^{\prime}}$ & $\frac{1}{\theta}$ & $\frac{\left.\theta^{2}+4 \theta+2\right)^{2}}{\theta\left(\theta^{2}+3 \theta+2\right)}$ \\
hazard rate function & $\theta$ & $\frac{\theta^{2}(1+x)}{1+\theta+\theta x}$ \\
mean residual life function $(\mathrm{m}(\mathrm{x}))$ & $\frac{1}{\theta}$ & $\frac{\theta+2+\theta x}{\theta(1+\theta+\theta x)}$ \\
\hline
\end{tabular}

\section{Generalizations of Lindley distribution}

Shanker et al. ${ }^{4}$ have comparative study on modeling of lifetime data using one parameter ${ }^{1}$ distribution and exponential distribution. Lindley distribution is not suitable for modeling data sets where there is large right tail or the tail approaches to zero at a faster rate. Such data sets are quite common in insurance problems and count data example in biology. This critical observation has motivated the authors to search for extensions of Lindley distribution. There exist many versions of generalized Lindley distribution and extensions. In this section, important among them are considered one by one.

\section{Two parameter Lindley distribution}

Shanker ${ }^{6}$ suggested a two parameter Lindley distribution (TPLD), of which the Lindley distribution is a particular case. There exist two forms type 1 and type 2 . The pdf of type 1 is defined as

$$
f(x ; \theta, \alpha)=\frac{\theta^{2}}{\alpha \theta+1}(\alpha+x) e^{-\theta x} ; x>0, \theta>0, \alpha \theta>-1
$$

When $\alpha=0$, it gives the Lindley distribution and $\alpha=0$, it gives the gamma $(2, \theta)$ distribution. The pdf is a mixture of exponential $\theta$ and gamma $(2, \theta)$ distribution where the mixing constant is $\frac{\alpha \theta}{\alpha \theta+1}$.

The cdf of type 1 TPLD is,

$$
F(x: \alpha, \theta)=1-\frac{1+\alpha \theta+\theta x}{\alpha \theta+1} e^{-\theta x} ; x>0, \theta>0, \alpha \theta>-1
$$

Then the corresponding failure rate function $\mathrm{h}(\mathrm{x})$ is

$$
h(x)=\frac{\theta^{2}(1+\alpha x)}{\theta+\alpha+\theta \alpha x} ; x>0, \theta>0
$$

The pdf of type 2 is defined as

$$
f(x ; \theta, \beta)=\frac{\theta^{2}}{\theta+\beta}(1+\beta x) e^{-\theta x} ; x>0, \theta, \beta,>0
$$

The cdf of type 2 TPLD is,

$$
F(x: \beta, \theta)=1-\frac{\theta+\beta+\beta \theta x}{\theta+\beta} e^{-\theta x} ; x>0, \theta>0, \beta>-\theta
$$

Then the corresponding failure rate function $\mathrm{h}(\mathrm{x})$ is

$$
h(x)=\frac{\theta^{2}(1+\beta x)}{\theta+\beta+\theta \beta x} ; x>0, \theta>0, \beta>-\theta
$$

The mean of TPLD is always greater than the mode, the distribution is positively skewed. The TPLD provides better fits than those by the one parameter Lindley distribution. ${ }^{7}$ Obtained a two parameter weighted Lindley distribution and studied its applications to survival data. ${ }^{8}$ Studied two parameter Lindley distribution for modeling waiting and survival data. ${ }^{9}$ Proposed an extended Lindley distribution which offers a more flexible model for lifetime data.

\section{Akash distribution}

The one parameter lifetime distribution with pdf

$$
f(x, \theta)=\frac{\theta^{3}}{\theta^{2}+2}\left(1+x^{2}\right) e^{-\theta x} ; x>0, \theta>0
$$

Suggested by Shanker ${ }^{10}$ is termed as Akash distribution. The pdf is a mixture of exponential $(\theta)$ and gamma $(3, \theta)$ distribution where the mixing constant $\frac{\theta^{2}}{\theta^{2}+2}$. The cdf of Akash distribution is 


$$
F(x)=1-\left[1+\frac{\theta x(\theta x+2}{\theta^{2}+2}\right] e^{-\theta x} ; x>0, \theta>0
$$

and the corresponding hazard rate function $\mathrm{h}(\mathrm{x})$ is

$$
h(x)=\frac{\theta^{2}(1+x)}{(\theta+1)+\theta x} ; x>0, \theta>0
$$

Akash distribution is over-dispersed (when $\mu>\sigma^{2}$ ), equidispersed (when $\mu>\sigma^{2}$ ) and under-dispersed (when $\mu>\sigma^{2}$ ). The hazard rate function of Akash distribution is an increasing function of $\mathrm{x}$ and $\theta$. Also it is better than Lindley and exponential distribution for modeling life time data from medical science and engineering. Shanker ${ }^{11}$ has introduced a quasi Akash distribution for modeling lifetime data and discussed its statistical properties and applications. $\mathrm{Also}^{11}$ have comparative study on lifetime data using one parameter Akash, Lindley and exponential distribution and showed that Akash distribution gives better fit in some of the data set than the other two distributions.

\section{Two parameter akash distribution}

Shanker ${ }^{12}$ proposed a two parameter Akash distribution (TPAD) with parameters $\theta$ and $\alpha$ is defined by its pdf

$$
f(x ; \theta, \alpha)=\frac{\theta^{3}}{\alpha \theta^{2}+2}\left(\alpha+x^{2}\right) e^{-\theta x} ; x>0, \theta>0, \alpha>0
$$

At $\alpha=1$, it gives the Akash distribution. The pdf of TPAD is a convex combination of exponential $(\theta)$ and gamma $(3, \theta)$ with mixing constant $\frac{\alpha \theta^{2}}{\alpha \theta^{2}+2}$. Its cdf

$$
F(x)=1-\left[1+\frac{\theta x(\theta x+2}{\alpha \theta^{2}+2}\right] e^{-\theta x} ; x>0, \theta>0, \alpha>0
$$

The corresponding failure rate function or hazard rate function $\mathrm{h}(\mathrm{x})$ is

$$
h(x)=\frac{\theta^{3}\left(\alpha+x^{2}\right)}{\theta x(\theta x+2)+\left(\alpha \theta^{2}+2\right)}
$$

TPAD gives better fit over exponential, Akash and Lognormal distributions. Shanker ${ }^{12}$ studied weighted Akash distribution for modelling lifetime data and observed that it gives better fit than several one parameter and two parameter lifetime distribution.

\section{Generalized Lindley distribution}

Zakerzadeh ${ }^{13}$ suggested a generalized Lindley distribution (GLD), of which the Lindley distribution is a particular case. The pdf is defined as

$$
f(x ; \alpha, \theta, \gamma)=\frac{\theta^{2}(\theta x)^{\alpha-1}(\alpha+\gamma x) e^{-\theta x}}{(\gamma+\theta) \Gamma(\alpha+1)} ; \alpha, \theta, \gamma, x>0
$$

That is this is a three parameter distribution. At $\alpha=\gamma=1$, it gives the Lindley distribution and $\gamma=0$, it gives the gamma $(\alpha, \theta)$ distribution .The pdf is a mixture of gamma $(\alpha, \theta)$ and $(\alpha+1, \theta)$ gammadistribution where the mixing constant is $\frac{\theta}{\gamma+\theta}$.

The cdf of Generalized Lindley distribution can be given only in terms of the incomplete gamma function when $\alpha$ is not an integer, the hazard function $\alpha$ could not be expressed in closed form.

\section{A new generalized Lindley distribution}

Ibrahim et al. ${ }^{14}$ proposed a new generalized Lindley distribution (NGLD) is obtained from a mixture of the gamma $(\beta, \theta)$ and gamma $(\beta, \theta)$ where the mixing constant is $\frac{\theta}{1+\theta}$. The pdf of NGLD is

$$
f(x ; \theta, \alpha, \beta)=\frac{1}{1+\theta}\left[\frac{\theta^{\alpha+1} x^{\alpha-1}}{\Gamma(\alpha)}+\frac{\theta^{\beta} x^{\beta-1}}{\Gamma(\beta)}\right] e^{-\theta x} ; \alpha, \theta>0 x>0
$$

The corresponding cdf is given by

$$
F(x ; \theta, \alpha, \beta)=\frac{1}{1+\theta}\left[\frac{\theta \gamma(\alpha, \theta x)}{\Gamma(\alpha)}+\frac{\gamma(\beta, \theta x)}{\Gamma(\beta)}\right] ; \alpha, \theta>0 x>0
$$

The hazard rate function $\mathrm{h}(\mathrm{x})$ is

$$
h(x)=\frac{\frac{1}{1+\theta}\left[\frac{\theta^{\alpha+1} x^{\alpha-1}}{\Gamma(\alpha)}+\frac{\theta^{\beta} x^{\beta-1}}{\Gamma(\beta)}\right] e^{-\theta x}}{1-\frac{1}{1+\theta}\left[\frac{\theta \gamma(\alpha, \theta x)}{\Gamma(\alpha)}+\frac{\gamma(\beta, \theta x)}{\Gamma(\beta)}\right]} ; \alpha, \theta>0 x>0
$$

The hazard rate function of GLD, is increasing for $\gamma>0$, bathtub shaped for $\gamma>0$ and $\gamma>0$, decreasing for $\alpha \leq 1$ and $\alpha=1$ . At $\alpha=1$ and $\beta=2$, the NGLD becomes Lindley distribution, For $\alpha=\beta=\lambda$, NGLD becomes gamma distribution with parameter $(\theta, \lambda)$ and at $\alpha=\beta=1$ it becomes exponential distribution with parameter $\theta$. Gupta ${ }^{15}$ studied generalized exponential distribution. The Lindley distribution has been generalized by different researchers including. ${ }^{16-21}$

\section{Power Lindley distribution} pdf

Ghitany et al. ${ }^{22}$ introduced Power Lindley distribution (PLD) with

$$
f(x ; \theta, \alpha)=\frac{\alpha \theta^{2}}{\theta+1}\left(1+x^{\alpha}\right) x^{\alpha-1} e^{-\theta x^{\alpha}} ; \theta, \alpha, x>0
$$

It is a new extension of Lindley distribution by considering the power transformation of the random variable $X=Y^{\frac{1}{\alpha}}$, where $\mathrm{Y}$ follows Lindley distribution. At $\alpha=1$ it reduces to Lindley distribution. It is a mixture of Weibull distribution $(2 \alpha, \theta)$ and generalized gamma distribution $(2 \alpha, \theta)$ with mixing proportion $\frac{\theta}{1+\theta}$.

The corresponding cdf is given by

$$
F(x ; \theta, \alpha)=1-\frac{\theta+1+\theta x^{\alpha}}{\theta+1} e^{-\theta x^{\alpha}} ; \theta, \alpha, x>0
$$

The associated hazard rate function is

$$
h(x)=\frac{\alpha \theta^{2} x^{\alpha-1}\left(1+x^{\alpha}\right.}{\left.\theta+1+\theta x^{\alpha}\right)} ; \theta, \alpha, x>0
$$

\section{Extended power Lindley distribution}

Alkarni ${ }^{23}$ suggested an Extended Power Lindley Distribution with parameters $\theta, \alpha$ and $\beta$ is defined by its pdf

$$
f(x ; \theta, \beta, \alpha)=\frac{\alpha \theta^{2}}{\theta+\beta}\left(1+\beta x^{\alpha}\right) x^{\alpha-1} e^{-\theta x^{\alpha}} ; \theta, \beta, \alpha, x>0
$$

It is a extension of type 2 TPLD by considering the power transformation of the random variable $X=Y^{\frac{1}{\alpha}}$, where $\mathrm{Y}$ follows 
type 2 TPLD. It is a mixture of Weibull distribution $(\alpha, \theta)$ and generalized gamma distribution $(2 \alpha, \theta)$ with mixing proportion $\frac{\theta}{\theta+\alpha}$. At $\alpha=\beta=1$. It gives the Lindley distribution, at $\alpha=1$ it gives TPLD, at $\beta=1$ it gives power distribution and at $\gamma=0$, it gives Weibull distribution.

The corresponding cdf is given by

$$
F(x ; \theta, \beta, \alpha)=1-\left(1+\frac{\theta \beta}{\theta+\beta} x^{\alpha}\right) e^{-\theta x^{\alpha}} ; \theta, \beta, \alpha, x>0
$$

The associated hazard rate function is

$$
h(x)=\frac{\alpha \theta^{2} x^{\alpha-1}\left(1+\beta x^{\alpha}\right)}{\theta+\beta+\beta \theta x^{\alpha}} ; \theta, \beta, \alpha, x>0
$$

$\mathrm{h}(\mathrm{x})$ is increasing for all $\theta>0$.

\section{Exponentiated power Lindley distribution}

Ashour ${ }^{24}$ suggested an Exponentiated Power Lindley Distribution with parameters $\theta, \alpha$ and $\beta$ is defined by its pdf

$$
f(x ; \theta, \beta, \alpha)=\frac{\alpha \theta^{2} \beta x^{\beta-1}}{\theta+1}\left(1+x^{\beta}\right) e^{-\theta x^{\beta}}\left[1-\left(1+\frac{\theta x^{\beta}}{\theta+1}\right) e^{-\theta x^{\beta}}\right]^{\alpha-1} ; \theta, \beta, \alpha, x>0
$$

For $\beta=1, \alpha=1$, and $\alpha=\beta=1$, It gives the generalized Lindley distribution, Power Lindley and Lindley distribution. The corresponding cdf is given by

$$
F(x ; \theta, \beta, \alpha)=\left[1-\left(1+\frac{\theta x^{\beta}}{\theta+1}\right) e^{-\theta x^{\beta}}\right]^{\alpha} ; \theta, \beta, \alpha, x>0
$$

The associated hazard rate function is

$$
\begin{aligned}
& h(x)=\frac{\alpha \theta^{2} \beta x^{\beta-1}}{\theta+1}\left(1+x^{\beta}\right) e^{-\theta x^{\beta}}\left[1-\left(1+\frac{\theta x^{\beta}}{\theta+1}\right] e^{-\theta x^{\beta}}\right]^{\alpha-1} S(x)^{-1} \\
& \text { Where }
\end{aligned}
$$

$$
S(x)=1-\left[1-\left(1+\frac{\theta x^{\beta}}{\theta+1}\right) e^{-\theta x^{\beta}}\right]^{\alpha}
$$

The coefficient of determination of Exponentiated Power Lindley Distribution is 0.975 , which is higher than the coefficient of determination of PLD, GLD, Lindley Distribution, Exponentiated exponential, modified Weibull and Weibull distributions. The Exponentiated Power Lindley Distribution is good model for life time data.

The power Lindley distribution with its inference was proposed by Ghitany et al..$^{22}$ and generalized by Liyanage ${ }^{18}$. Ghitany et al. ${ }^{25}$ discussed the estimation of the reliability of a stress-strength system from power Lindley distribution.

\section{Inverse Lindley distribution}

Sharma et al. $^{26}$ investigated inverted version of the Lindley distribution that has upside down bathtub shaped failure rate. The inverse Lindley distribution (ILD) is defined by the following pdf

$$
f(x ; \theta)=\frac{\theta^{2}}{1+\theta}\left(\frac{1+x}{x^{3}}\right) e^{\frac{-\theta}{x}} ; x, \theta>0
$$

ILD is a mixture of inverse exponential distribution and special case of inverse gamma distribution with mixing constant $\frac{\theta}{1+\theta}$

The cdf of ILD is

$$
F(x ; \theta,)=\left[1+\frac{\theta}{1+\theta} \frac{1}{x}\right] e^{\frac{-\theta}{x}} ; x, \theta>0
$$

The hazard rate function is

$$
h(x)=\frac{\theta^{2}(1+x)}{x^{2}\left[\theta+x(1+\theta)\left(e^{\frac{-\theta}{x}}-1\right)\right]}
$$

The ILD fits quit well the data of survival of Head and Neck cancer patients. A new upside-down bathtub-shaped hazard rate model for survival data analysis was proposed by Sharma et al. ${ }^{27}$ by using transmuted Rayleigh distribution. Sharma et al ${ }^{26}$ introduced the ILD as a one parameter model for a stress-strength reliability model. Alkarni ${ }^{28}$ proposed three parameter ILD with application to maximum flood level data. Also ${ }^{29}$ studied the extension of ILD.

\section{Truncated Lindley distribution}

Ahmed et al. ${ }^{30}$ introduced truncated version of Lindley Distribution. The truncated distribution is used where a random variable is restricted to be observed on some range. The truncated versions of the Lindley distribution, named as the upper truncated Lindley (UTL), lower truncated Lindley (LTL), double truncated Lindley (DTL) distributions are introduced.

The pdf of DTL distribution is denoted by $g_{D}(X ; \theta)$,

$$
g_{D}(X ; \theta)=\frac{\theta^{2}}{1+\theta} \frac{(1+x) e^{-\theta x}}{F(\zeta ; \theta)-F(v ; \theta)} ; 0 \leq v \leq x \leq \zeta<\infty
$$

At $\mathrm{v}=0$ and $\zeta \rightarrow \infty$, it reduces to baseline model here it is Lindly distribution, at $\mathrm{v}=0$, it gives the upper truncated distribution of the Lindley distribution and at $\zeta \rightarrow \infty$, it is called the lower truncated distribution of the Lindly distribution.

The pdf of UTL distribution is,

$$
g_{U}(x ; \theta)=\frac{\theta^{2}(1+x) e^{-\theta(x-\zeta)}}{(1+\theta)\left(e^{\theta \zeta}-1\right)-\theta \zeta} ; 0 \leq x \leq \zeta
$$

when $\theta<1, g_{U}(x)$ is uni-modal and mode values is $M_{0}=(1-\theta) / \theta$ and the corresponding hazard rate function at $\mathrm{t}$ is

$$
h(t)=\frac{\theta^{2}}{1+\theta} \frac{(1+t) e^{-\theta t}}{F(\zeta ; \theta)-F(t ; \theta)} ; 0 \leq t \leq \zeta
$$

The hazard rate function of UTL distribution is increasing in $\mathrm{x}$ and $\theta$.

The pdf of LTL distribution is

$$
g_{L}(x ; \theta)=\frac{\theta^{2}(1+x) e^{-\theta(x-v)}}{1+\theta+\theta v ;} ; 0 \leq v \leq x
$$

The truncated distributions can be quit effectively used to model the real problems and so we can use the truncated Lindley distributions in various fields including engineering, medical, finance and demography where such type of truncated data are commonly encountered. Among the three truncated versions DTL is more effective than UTL and LTL, also UTL is more effective than LTL.

Many researchers propose the truncated versions of the usual statistical distributions including, Ahmed et al. ${ }^{31}$ discussed the application of the truncated version of the Birnbaum-Saunders (BS) distribution to improve a forecasting actuarial model and particularly. Zaninett ${ }^{32}$ discussed the application of the truncated Pareto distribution to the statistical analysis of masses of stars and of diameters of asteroids for modelling data from insurance payments that establish a 
deductible. Zhang ${ }^{33}$ studied truncated Weibull distribution.

\section{Discrete Lindley distribution}

Deniz $^{34}$ proposed the discrete Lindley distribution (DLD). It is obtained by discrediting the continuous failure model of the Lindley distribution. The probability mass function (pmf) of DLD is

$$
\begin{gathered}
f(x)=P(X=x)=\frac{\lambda^{x}}{1-\log \lambda}\left[\lambda \log \lambda+(1-\lambda)\left(1-\log \lambda^{x+1}\right)\right] ; x=0,1, \ldots \\
\text { corresponding cdf is } \\
F(x)=\frac{1-\lambda^{x+1}+\left((2+x) \lambda^{x+1}-1\right) \log \lambda}{1-\log \lambda}
\end{gathered}
$$

and the hazard rate function is

$$
h(x)=\frac{\lambda \log \lambda+(\lambda-1)\left(\log \lambda^{x+1}\right)-1}{1-(1+x) \log \lambda}
$$

The mean and variance of DLD is increase with $\lambda$ and overdispersed, therefore, more flexible than the Poisson distribution to model actuarial data that commonly include the over-dispersion phenomenon. It has an increasing hazard rate and it is unimodal. For large values of the parameter $\lambda$, the mode moves to the right, showing a great versatility. Also if $\mathrm{X}$ follows a continuous Lindley distribution with parameter $\theta>0$ then the random variable $\mathrm{Y}=[\mathrm{X}]$ follows a DLD with parameter $\lambda=e^{-\theta}$, here [.] denotes the integer part.

The researchers who were discretise various continuous distributions including. ${ }^{35-40}$

\section{Poisson Lindley distribution}

A mixture distribution is the probability distribution of a random variable that is derived from a collection of other random variables. A compound distribution resembles in many ways the original distribution that generated it, but typically has greater variance, and often heavy tails as well. Any probability distribution on $[0, \infty)$ can function as the mixing distribution for a poisson mixture.

\section{Discrete poisson Lindley distribution}

The discrete Poisson Lindley distribution (DPLD) is the Poisson distribution compounded with Lindley distribution and is proposed by Sankaran ${ }^{41}$.

The pdf of DPLD is,

$$
f(x)=P(X=x)=\frac{\theta^{2}(x+\theta+2)}{(\theta+1)^{x+3}} ; x=0,1,2 \ldots, \theta>0
$$

The DPLD is over dispersed, so it can be used in fields like biological science and medical science. In the field of genetics and ecology DPLD gives much closer fit than Poisson distribution and thus it can be considered as an important tool for modeling data in these field.

Ghitany $^{42}$ discussed the estimation methods for the DPLD and its applications. The DPLD has been generalized by many researchers. Shanker ${ }^{43}$ studied a two parameter Poisson-Lindley distribution by compounding Poisson distribution with a two parameter Lindley distribution. Shanker ${ }^{44}$ obtained a new quasi Poisson-Lindley distribution by compounding Poisson distribution with a new quasi Lindley distribution introduced by Shanker et al ${ }^{45}$ obtained a discrete two parameter Poisson Lindley distribution by mixing Poisson distribution with a two parameter Lindley distribution. Also Shanker ${ }^{46}$ studied Poisson-Lindley distribution and its application to biological science.

\section{Discrete poisson-Akash distribution(DPAD)}

Shanker ${ }^{47}$ proposed DPAD. The pdf of Poisson mixture of Akash distribution is

$$
f(x)=\frac{\theta^{3}}{\theta^{2}+2} \frac{x^{2}+3 x+\left(\theta^{2}+2 \theta+3\right)}{(\theta+1)^{x+3}} ; x=0,1,2 \ldots, \theta>0
$$

DPAD has an increasing hazard rate and unimodal and always over-dispersed thus it is a suitable model for count data which are over-dispersed. DPAD gives much closer fit than Poisson distribution and DPLD in almost all cases, DPAD has some flexibility over DPLD.

Shanker et al. ${ }^{48}$ discussed Poisson-Akash Distribution and its Applications. Shanker ${ }^{49}$ has also introduced size based and zero truncated version of Poisson Akash distribution and studied their properties.

\section{Transmuted Lindley distribution}

Merovci ${ }^{50}$ Proposed Transmuted Lindley Distribution. The pdf of Transmuted Lindley Distribution is,

$$
f(x)=\frac{\theta^{2}}{1+\theta}(1+x) e^{-\theta x}\left(1-\lambda+2 \lambda \frac{\theta+1+\theta x}{\theta+1} e^{-\theta x}\right) ; x>0, \theta>0
$$

The transmuted Lindley distribution is an extended model to analyze more complex data and it generalizes some of the widely used distributions, at $\lambda=0$ it gives Lindley distribution. The corresponding cdf is,

$$
F(x)=\left(1-\frac{\theta+1+\theta x}{\theta+1} e^{-\theta x}\right)\left(1+\frac{\theta+1+\theta x}{\theta+1} e^{-\theta x}\right)
$$

The hazard rate function is

$$
h(x)=\frac{\theta^{2}(1+x)\left(1-\lambda+2 \lambda \frac{\theta+1+\theta x}{\theta+1} e^{-\theta x}\right)}{(1+\theta+\theta x)\left(\lambda-1-\lambda \frac{\theta+1+\theta x}{\theta+1} e^{-\theta x}\right)}
$$

From this if $\lambda=1$, the hazard rate is decreasing. Aryal ${ }^{51}$ studied Transmuted Weibull distribution and its properties.

\section{Wrapped Lindley distribution}

A wrapped probability distribution is a continuous probability distribution that describes data points that lie on a unit $\mathrm{n}-$-sphere. The cases of wrapped Lindley distribution have been studied extensively by Joshi ${ }^{52}$. The wrapped Lindley (WL) random variable is defined as $\theta=X(\bmod 2 \pi)$, such that for $\theta \in[0,2 \pi]$, the pdf is given by

$$
g(\theta)=\frac{\lambda^{2}}{1+\lambda} e^{-\lambda \theta}\left[\frac{1+\theta}{1-e^{-2 \pi \lambda}}+\frac{2 \pi e^{-2 \pi \lambda}}{\left(1-e^{-2 \pi \lambda}\right)^{2}}\right], \theta \in[0,2 \pi), \lambda>0
$$

The random variable $\theta$ having wrapped Lindley distribution is denoted by $\theta \sim W L(\lambda)$. The cdf of WL is

$$
G(\theta)=\frac{1}{1-e^{-2 \pi \lambda}}\left(1-e^{-\lambda \theta}-\frac{\lambda \theta}{1+\lambda} e^{\lambda \theta}\right)-\frac{2 \pi \lambda}{1+\lambda}\left(1-e^{\lambda \theta}\right)\left(\frac{e^{-2 \pi \lambda}}{1-e^{-2 \pi \lambda}}\right) \theta \in[0,2 \pi), \lambda>0
$$

Joshi $^{52}$ showed that wrapped Lindley distribution give good fit to the data data set (orientations of 76 turtles after laying eggs and is given in Table $1\left(\mathrm{Rao}^{53}\right)$ than wrapped exponential distribution.

\section{Conclusion}

This paper studied the well-established and widely used Lindley distribution and its generalizations. The variety of generalizations 
or parameters induction can be used to handle various real data sets with complex structure. These models will be useful for constructing probability models and may help the development of new classes from the Lindley distribution in future.

\section{Acknowledgements}

None.

\section{Conflict of interest}

The author declares that there in none of the conflicts.

\section{References}

1. Lindley DV. Fiducial distributions and Bayes theorem. Journal of the Royal Statistical Society A. 1958;20(1):102-107.

2. Ghitany ME, Atieh B, Nadarajah S. Lindley distribution and its Applications. Mathematical Computation and Simulation. 2008;78(4):493-506.

3. Mazucheli J, Achcar JA. The Lindley distribution applied to competing risks lifetime data. Comput Methods Programs Biomed. 2011;104(2):188-192.

4. Shanker R, Hagos F, Sujatha S. On modeling of Lifetimes data using exponential and Lindley distributions. Biometrics \& Biostatistics International Journal. 2015;2(5):1-9.

5. Shanker R, Mishra A. A quasi Lindley distribution. African Journal of Mathematics and Computer Science Research. 2013;6(4):64-71.

6. Shanker R, Mishra A. A two parameter Lindley distribution. Statistics in transition new series. 2013;14(1):45-56.

7. Ghitany ME, Al-Qallaf F, Al Mutairi DK, et al. A two parameter weighted lindley distribution and its applications to survival data. Mathematics and computers in Simulation. 2011;81(6):1190-1201.

8. Shanker R, Sharma S, Shanker R. A two parameter Lindley distribution for modeling waiting and survival time series data. Applied Mathematics. $2013 ; 4: 363-368$.

9. Bakouch HS, Al Zahrani BM, Al Shomrani AA, et al. An extended Lindley distribution. Journal of the Korean Statistical Society. 2012;41(1):75-85.

10. Shanker R. Akash distribution and its applications. International Journal of probability and statistics. 2015;4(3):65-75.

11. Shanker R. A quasi Akash distribution. Assam Statistical Review. 2016;30(1):135-160.

12. Shanker R, Shukla KK. Weighted Akash distribution and its application to model lifetime data. International Journal of Statistics. 2016;39(2):1138-1147.

13. Zakerzadeh H, Dolati A. Generalized Lindley distribution. Journal of Mathematical Extension. 2009;3(2):13-25.

14. Ibrahim E, Merovci F, Elgarhy M. A new generalized Lindley distribution. Mathematical Theory and Modeling. 2013;3(13):30-47.

15. Gupta RD, Kundu D. Generalized exponential distribution. Australian and New Zealand Journal of Statistics. 1999;4:1-23.

16. Abouammoh AM, Alshangiti AM, Ragab IE. A new generalized Lindley distribution. Journal of Statistical computation and simulation. 2015;85(18):3662-3678.
17. Elbatal I, Merovi F, Elgarhy M. A New generalized Lindley distribution. Mathematical Theory and Modelling. 2013;3(13):30-37.

18. Liyanage G, Parai M. The generalized power Lindley distribution with its applications. Asian journal of mathematics and applications. 2014;73:331-359.

19. Nadarajah S, Bakouch HS, Tahmasbi R. A generalized Lindley distribution. Sankhya series B. 2011;73(2):331-359.

20. Oluyede BO, Yang T. A New class of generalized Lindley distribution with its applications. Journal of Statistical computation and simulation. 2015;85(10):2072-2100.

21. Pararai M, Warahena Liyanage G, Oluyede BO. A New Class of Generalized Power Lindley Distribution with Applications to Lifetime Data. Theoretical Mathematics \& Applications. 2015;5(1):53-96.

22. Ghitany ME, Al Mutairi DK, Balakrishnan N, et al. Power Lindley distribution and associated inference. Computational statistics and data analysis. 2013;6:20-33.

23. Alkarni SH. Extended power Lindley distribution:A new statistical model or non monotone survival data. European Journal of statistics and probability. 2015;3(3):19-34.

24. Ashour S, Eltehiwy M. Exponentiated power Lindley distribution. Journal of Advanced Research. 2015;6(6):895-905.

25. Ghitany ME, Al Mutairi DK, Aboukhamseen SM. Estimation of the reliability o a stress-strength system from power Lindley distribution. Computational statistics and data analysis. 2015;44(1):118-136.

26. Sharma VK, Singh SK, Singh U, et al. The inverse Lindley distribution: A stress reliability model with application to head and neck cancer data. Journal of Industrial and Production Engineering. 2015;32(3):162-173.

27. Sharma VK, Singh SK, Singh U. A new upside-down bathtub shaped hazard rate model for survival data analysis. Applied Mathematics and Computation. 2014;239:242-253.

28. Alkarni SH. Extended inverse Lindley distribution: properties and application. Springer-Plus. 2015;4:1-13.

29. Sharma VK, Khandelwal P. On the extension of inverse Lindley distribution. Journal of Data Science. 2017;15(2):205-220.

30. Singh SK, Singh U, Sharma VK. The Truncated Lindley DistributionInference and Application. Journal of Statistics Applications and Probability. 2014;3(2):219-228.

31. Ahmed SE, Castro Kuriss C, Flores E, et al. A truncated version of the birnbaum-saunders distribution with an application in financial risk. Pakistan Journal of Statistics. 2010;26(1):293-311.

32. Zaninetti L, Ferraro M. On the truncated Pareto distribution with applications. Central European Journal of Physics. 2008;6:1-6.

33. Zhang T, Xie M. On the upper truncated Weibull distribution and its reliability implications. Reliability Engineering and System Safety. 2011;96(1):194-200.

34. Deniz E, Ojeda E. The discrete Lindley distribution: Properties and application. Journal of Statistical Computation and Simulation. 2011;81(11):1405-1416.

35. Al Huniti A, Al Dayian G). Discrete burr type-iii distribution. American Journal of Mathematics and Statistic. 2012;2(5):145-152.

36. Chakraborty S, Chakravarty D. Discrete gamma distributions: Properties and parameters estimations. Communication in Statistics Theory and Methods. 2012;41(18):3301-3324. 
37. Krishna H, Pundir P. Discrete burr and discrete pareto distributions. Statistical Methodology. 2009;6(2):177-188.

38. Nakagawa T, Osaki S. The discrete Weibull distribution. IEEE Transactions on Reliability. 1975;24(5):300-301.

39. Roy D. Discrete normal distribution. Communication in Statistics Theory and Methods. 2003;32(10):1871-1883.

40. Roy D. Discrete rayleigh distribution. IEEE Transactions on Reliability. 2004;532):255-260.

41. Sankaran M. The discrete Poisson-Lindley distribution. Biometrics 1970;26(1):145-149.

42. Ghitany ME, Al Mutairi DK. Estimation Methods for the discrete Poisson-Lindley distribution. Journal of Statistical Computation and Simulation. 2009;79(1):1-9.

43. Shanker R, Mishra A. A two-parameter Poisson-Lindley distribution International Journal of Statistics and Systems. 2014;9(1):79-85.

44. Shanker R, Tekie AL. A new quasi Poisson-Lindley distribution. International Journal of Statistics and Systems. 2014; 9(1):

45. Shanker R, Sharma S, Shanker R. A Discrete two-Parameter Poisson Lindley Distribution. Journal of Ethiopian Statistical Association. 2012;21:15-22.
46. Shanker R, Fesshaye H. On Poisson-Lindley Distribution and Its Applications to Biological Sciences. Biometrics and Biostatistics International Journal. 2015;2(4):1-5.

47. Shanker R. The discrete Poisson-Akash distribution. International Journal of probability and statistics. 2017;6(1):1-10.

48. Shanker R, Fesshaye H, Tesfazghi T. On Poisson-Akash Distribution and its Applications. Biometrics and Biostatistics International Journal. 2016;3(6):1-6.

49. Shanker R. Size biased Poisson-Akash distribution and its applications. International Journal of probability and statistics. 2014;7(6):289-297.

50. Merovci F. Transmuted Lindly Distribution. Int J Open Problems Compt Math. 2013;6(2):63-72.

51. Aryal GR, Tsokos CP. Transmuted Weibull Distribution: A Generalization of the Weibull probability distribution. European journal of pure and applied mathematics. 2011;4(2):89-102.

52. Joshi S, Jose KK. Wrapped Lindley distribution. Communications in Statistics-Theory and Methods. 47(5):1013-1021.

53. Rao JS, Sen Gupta A. Topics in circular statistics. USA: New York: World Scientific; 2001. 322 p. 